the absence of physical factors. This applies - as in any other branch of medicine-to the decision whether or not to treat by hypnosis.

\section{Consulting Rooms}

Maurice SilvermaN

Beardwood Hospital

Preston New Road

Blackburn BB2 7 BG

\section{References}

MACKETT, J. (1985) Hypnotherapy for subfertility: report of two cases. Proceedings of the British Society of Medical and Dental Hypnosis, 6, 20-21.

Whorwell, P. J., Prior, A. \& Faragher, E. B. (1984) Control trial of hypnotherapy in the treatment of severe refractory irritable bowel syndrome. Lancet, ii, 1232-1234.

- - \& Colgam, S. M. (1987) Hypnotherapy in severe irritable bowel syndrome: further experience. Gut, 28, 423-435.

\section{Reply}

The main aim of my article was to outline the current situation of hypnosis and its possible application in psychiatry, not to discuss details of case reports and possible further application in general practice.

I am sad that Dr Silverman misunderstood my statement of "substitute to treat the untreatable". Hypnotherapy has always been misused and has unfortunately been applied as a main therapy for malignant disorders and even HIV infection which currently cannot be treated by any known conventional methods. Hypnotherapy has also been misused to treat disorders which may be treated by surgery. Therefore, hypnotherapy must not be considered as an alternative or "substitute" or secondline therapy for any disorder which is currently untreatable by conventional or known methods.

I am surprised that Dr Silverman thinks that organic abnormality should not be taken into account as he states in his final paragraph "this may or may not be true". To elaborate the point further Dr Silverman quotes the paper by Mackett (1985) "the use of hypnosis in infertility without any organic basis". How and who decided about the organic basis and why did they not use people with "organic basis"?

I have tried to keep the paper as brief as possible with a view to encouraging psychiatrists in my venture to establish a 'Hypnotherapy Special Interest Group' within the Royal College of Psychiatrists, about which I have already written to the President.

University of Leicester V. M. MatheW Clinical Sciences Building

Leicester Royal Infirmary

PO Box 65

Leicester LE2 7LX
User satisfaction with a psychiatric day hospital

\section{DeAR SIRS}

The Mental Illness Key Area Handbook from the Department of Health emphasises that central to the creation of "appropriate and responsive services" is involvement of users "with the most severe mental illnesses and the most longstanding health and social problems". We wish to report our recent study which examined the pattern of need of patients attending a psychiatric day hospital in an inner city area of high social deprivation.

We used a structured interview on a comprehensive patient sample recruited across all hospital working days and opening hours: the sample (23) was representative of all 96 patients on the attendance register. Day patients tended to be single, middleaged, isolated, with mainly psychotic illness, had an approximately equal sex distribution and ethnic minorities composition similar to the general population. In-patient admissions were about three times the length of other catchment area patients, and social disabilities were severe according to World Health Organisation criteria.

Twenty-one $(91 \%)$ of the patient sample named their key worker. High levels of satisfaction were reported for professionals that patients had most access to and contact with: $74 \%$ of patients rated nurses as "helpful" or "very helpful", while similar ratings for occupational therapists were $96 \%$. Psychiatrists' ratings were lower (consultants: $30 \%$, registrars: $52 \%$ ). The reported helpfulness of psychologists and social workers depended on their accessibility. Average group attendance was ten patients (range 4 to 15); regression analysis showed a consistent level of satisfaction of $71 \%$ across selfselecting groups $(n=15, P<0.0001)$ of all sizes. Most patients $(74 \%)$ believed their mental health was benefitting from attendance, and over half (52\%) said their care was strengthened by sharing it with others.

Our initial, anonymous, self-completed questionnaire (a widely used method) failed to obtain any useful information from this patient population. However, using our structured interview we identified specific areas in which care was subsequently improved. We would stress that in-depth interviewing requires many manhours, including negotiation and consultation with, and feedback to, staff and users. In our opinion, all staff should be involved in planning the audit, and interpretation of the implications for change.

JOAN RUTHERFORD

The Ridgewood Centre

Old Bisley Road, Frimley

Surrey GU16 5QE 
Maudsley Hospital

Denmark Hill

London SE5 18AZ

References are available on request to Dr Rutherford.

\section{Working in a child guidance clinic}

DeAR SIRS

In my previous job I worked as a registrar in a child guidance clinic; before that I had always worked in acute adult psychiatry. This clinic and its practice was new in many ways: it was outside a hospital, there was less emphasis on the medical model and pharmacological treatment, less use of a classification system and less clear sense of hierarchy and boundaries between the disciplines. This apparent lack of structure was not reflected in a typical day: all contact with patients was arranged by appointments; the diary was fully booked hour after hour, weeks in advance; and there was no pager to interfere with sessions (what a delight).

As a newcomer it was hard to define one's own (and others') identity in, and particular contribution to, the service. Treating patients within their social and emotional environment (the family) was difficult and challenging. As the biological model of mental illness did not predominate, problems were understood and treated in psychological terms. These views were very much fostered by the non-medical colleagues and the particular interests of the psychiatrist.

Working in a multidisciplinary system where there is less emphasis on the medical model and hierarchy broadened my perspective. A constant appeal to defining and identifying the special abilities of myself and my colleagues made me aware of the available resources and their contributions to the management of a case.

I believe that every trainee should be strongly encouraged to gain experience in child and family psychiatry in order to enhance self understanding. This will then be reflected in treatment. I also propose that every junior doctor should at least have one supervised psycho-analytic experience. I would recommend an early introduction into such a job provided there is adequate supervision.

I wonder if there are trainees and consultants who share my views?

Charing Cross Psychiatric Rotation Scheme

JAN HERMSEN

Silkstream Unit

Colindale Hospital

London NW9

\section{Crisis team in South Lewisham}

DeAR SIRS

The Crisis Team in South Lewisham has been in operation since January 1992 and consists of an approved social worker and community psychiatric nurse, with input from a consultant psychiatrist.

The possibility of the CPN being able to prescribe medication from an agreed list of drugs to a client in crisis, to alleviate acute distress and possibly prevent hospital admission, was examined. To obtain their views on this proposal a questionnaire was sent to GPs, consultant psychiatrists and CPNs; 17 out of $30 \mathrm{GPs}$, 12 out of 16 psychiatrists and 13 out of 20 CPNs replied.

Fifteen GPs agreed that CPNs should prescribe in crisis situations from a negotiated list but suggested that GPs be made aware if a CPN prescribes. Nine psychiatrists felt that it was a 'positive' step but some felt it may not be useful; 11 CPNs agreed with the principle.

As to whether CPNs should also prescribe as part of on-going care, ten GPs, seven psychiatrists, and nine CPNs said yes and two GPs, five psychiatrists, and three CPNs, no.

Thirteen GPs, seven psychiatrists and 11 CPNs had confidence in nurses prescribing in crises but with such comments as "with adequate training and supervision" and with the suggestion that crises are the worst time for the nurse prescribing. Also, concerns about possible drug interactions, poor communication and the blurring of roles were raised. However, positive factors could be saving of time in crises thus relieving acute distress, increasing the effectiveness of community nurses, and greater flexibility. When asked who should take responsibility for nurse prescribing, 14 GPs and six psychiatrists felt that the psychiatrist should while eight CPNs felt that nurses should take their own responsibility.

The range of medication that $C P N$ s could prescribe was considerable. GPs felt that tranquillisers, antidepressants and hypnotics should be included but the psychiatrists and CPNs did not include antidepressants. The time limit placed on prescriptions ranged from one dose to several days, i.e. to cover a weekend. On what precautions were needed for such a policy to work, suggestions included adequate training, support and regular updating. As to whether a CPN who had known clients for years should be able to alter times and dosages of medication, most GPs (12) said yes, as did seven psychiatrists and 13 CPNs.

Psychiatrists and CPNs on the whole are therefore in favour of a CPN being able to "prescribe" from a small negotiated list of drugs in crisis situations. As long as the psychiatrist took responsibility for the nurse prescribing they would have confidence in the CPN to carry out such a function.

Communication between CPN, psychiatrist and GP was felt to be the most vulnerable point. So, for 